\title{
Transcriptome analysis in mouse tumors induced by Ret-MEN2/FMTC mutations reveals subtype-specific role in survival and interference with immune surveillance
}

\author{
D Engelmann ${ }^{1}$, D Koczan², P Ricken ${ }^{1}$, U Rimpler ${ }^{1}$, J Pahnke ${ }^{3}, Z$ Li $^{1}$ \\ and B M Pützer ${ }^{1}$
}

\author{
${ }^{1}$ Department of Vectorology and Experimental Gene Therapy, Biomedical Research Center, ${ }^{2}$ Institute for Immunology, University of \\ Rostock, D-18055 Rostock, Germany \\ ${ }^{3}$ Department of Neurology, University of Rostock, D-18147 Rostock, Germany \\ (Correspondence should be addressed to B M Pützer; Email: brigitte.puetzer@med.uni-rostock.de)
}

\begin{abstract}
Activating mutations in the Ret proto-oncogene are responsible for occurrence of multiple endocrine neoplasia (MEN) type $2 \mathrm{~A}$ and $2 \mathrm{~B}$, and familial medullary thyroid carcinoma (FMTC). A striking genotype-phenotype correlation between the mutated RET codon and clinical manifestation implies that tumorigenesis is conditioned by the type of mutation. We investigated gene expression profiles between and within distinct MEN2 subtypes through whole-genome microarray analysis in tumors induced by NIH-3T3 cells transformed with defined RET-MEN2A (C609Y, C634R), MEN2B, (A883F, M918T), and FMTC (Y791F) mutations. Expression profiling identified a statistically significant modification of 1494 genes, 628 down- and 866 upregulated in MEN2B compared with MEN2A/FMTC tumors. By contrast, no obvious alterations were observed among individual MEN2B and MEN2A type mutations, or between MEN2A and FMTC. Functional clustering of differential genes revealed RET-MEN2B specific upregulation of genes associated with novel growth and survival pathways. Intriguingly, RET-MEN2A/FMTC-specific tumors were characterized by a considerable number of genes involved in the host antitumor immune response via stimulation of natural killer/T-cell proliferation, migration, and cytotoxicity, which were completely absent in RET-MEN2B related cancers. QPCR on tumors versus cultured NIH-RET cell lines demonstrated that they are largely attributed to the host innate immune system, whereas expression of CX3CL1 involved in leukocyte recruitment is exclusively RET-MEN2A/FMTC tumor cell dependent. In correlation, massive inflammatory infiltrates were apparent only in tumors carrying MEN type 2A/FMTC mutations, suggesting that RET-MEN2B receptors specifically counteract immune infiltration by preventing chemokine expression, which may contribute to the different clinical outcome of both subtypes.
\end{abstract}

Endocrine-Related Cancer (2009) 16 211-224

\section{Introduction}

The Ret gene on chromosome 10q11.2 was originally identified as an oncogene activated by DNA rearrangement that encodes a transmembrane receptor of the tyrosine kinase family (Takahashi et al. 1985). RET serves as a functional receptor for neurotrophic factors of the glial cell-line-derived neurotrophic factor (GDNF) family: GDNF, neurturin, artemin, and persephin. Binding to and activation of RET occurs via co-receptors that are designated as GDNF-family receptors $\alpha 1-4$. Ligand stimulation leads to the activation of the RET receptor by dimerization and subsequent autophosphorylation of intracellular tyrosine residues. These, in turn, serve as docking sites for a number of interacting molecules activating downstream signal transduction pathways. Gene knockout studies revealed that GDNF/RET signaling plays a crucial role in the development of the enteric nervous system and the kidney (Drosten \& Pützer 2006, de Groot et al. 2006). 
Autosomal dominant gain of function mutations in the Ret proto-oncogene have been identified as the key cause for the development of multiple endocrine neoplasia type 2 (MEN2), which can be further divided into two distinct clinical manifestations MEN2A, MEN2B and familial medullary thyroid carcinoma (FMTC; Lakhani et al. 2007). They render the RET receptor constitutively active and considerably influence the disease phenotype, severity, and the age of onset. Patients with MEN2A not only develop medullary thyroid carcinoma (MTC), but also pheochromocytoma $(50 \%)$ and parathyroid hyperplasia or adenoma (20-30\%). MEN2B has the same features as MEN2A, but with earlier onset and developmental abnormalities such as mucosal neuromas, intestinal ganglioneuromas, ocular and skeletal abnormalities (marfanoid habitus). FMTC is characterized by the incidence of MTC only. From all three syndromes, MEN2B cancer tends to be more aggressive and leads to death from metastatic disease in young children. By contrast, familial MTC behaves in the least aggressive fashion, and, although lymph node metastases are frequent, the disease usually follows an indolent course, and virtually never results in death. Mutations identified in $>98 \%$ of MEN2A patients affect one of six cysteine residues in the cysteine-rich region at codons 609, 611, 618, 620 (exon 10), 630, or 634 (exon 11) and cause ligand-independent homodimerization through covalent intermolecular disulfide bonds, resulting in subsequent constitutive activation of the RET kinase, which, in turn, leads to permanent downstream signaling. Approximately $87 \%$ of MEN2A mutations affect codon 634. By contrast, mutations found in MEN2B patients affect residues in the tyrosine kinase domain and activate the RET receptor in its monomeric state, thereby changing the substrate specificity toward other cellular substrates and downstream signaling pathways. In addition, increased auto-phosphorylation of tyrosine 1062 has been described. MEN2B is primarily associated with a single missense mutation of codon 918 (M918T), which is detectable in more than $90 \%$ of MEN2B patients. A smaller number of MEN2B cases contains mutations at codon 883 (A883F). Mutations identified in FMTC patients (for example at codons 790, 791, or 844 ) are found in the cysteine-rich region as well as in the tyrosine kinase domain, and lead to low-level activation of the RET kinase corresponding to the indolent penetrance phenotype of FMTC; reviewed in de Groot et al. (2006), Plaza-Menacho et al. (2006).

The molecular link of different Ret mutations to distinct clinical MEN2 syndromes is still largely unknown. To further elucidate the mechanism by which alterations in a major oncogene result in a disparate biology of closely related cancer syndromes, in particular a higher aggressiveness of MEN type-2B mutations, we performed a whole-genome microarray expression analysis using a defined in vivo model of NIH-3T3 mouse fibroblasts expressing various RETMEN2A (C609Y and C634R), RET-MEN2B (A883F and M918T) and FMTC (Y791F) mutations.

\section{Materials and methods}

\section{Cell lines and tumor samples}

NIH-3T3 fibroblast cell lines stably expressing the long $\mathrm{RET}^{51}$ isoform carrying the MEN2A mutations C609Y and C634R, Y791F (FMTC), and the MEN2B-derived mutations $\mathrm{A} 883 \mathrm{~F}$ and $\mathrm{M} 918 \mathrm{~T}$ have been described previously (Miše et al. 2006). Cultures were maintained in DMEM (Life Technologies) supplemented with $10 \%$ fetal bovine serum (PAA Laboratories, Coelbe, Germany) and 1\% penicillin G/streptomycin sulfate (Life Technologies). Tumors were established by s.c. injection of $10^{6}$ NIH-RET(MEN2A), and NIHRET(MEN2B) cells into the hind flank of 6- to 8-weekold NMRI/nude mice. Tissue samples were collected from all animals 3 weeks after injection, weighed, snap-frozen in liquid nitrogen immediately after resection, and stored at $-80{ }^{\circ} \mathrm{C}$. All mice experiments were approved by and conducted in accordance with institutional animal care guidelines.

\section{RNA isolation and microarray hybridization}

Total RNA from frozen tumor tissue was prepared by standard methods. Five microgram total RNA was used to prepare biotinylated cRNA targets, which were hybridized to Mouse 4302.0 GeneChips according to the supplier's instructions (Affymetrix, Santa Clara, CA, USA). Hybridization and washing of gene chips was performed on an Affymetrix GeneChip Hybridization Oven 640 and Fluidics Station 450 in compliance with standard procedures. Microarrays were analyzed by laser scanning (Affymetrix GeneChip Scanner 3000).

\section{Microarray data processing and analysis}

Three independent GeneChip expression analyses were performed for each mutation. Background-corrected signal intensities were determined and processed using MAS5 function of the R/Bioconductor affy package (www.r-project.org/www.bioconductor.org). All calculations including normalization of microarray data, statistical tests, clustering, and further filtering methods were accomplished by the gene expression 
analysis software GeneSpring GX 9.0 (Agilent Technologies, Wilmington, DE, USA). Genes whose transcripts were not detected in any of the investigated mutations were excluded from statistical analysis to reduce the number of false positive genes. To determine differentially expressed genes, expression data were grouped according to MEN2B and MEN2A/ FMTC mutations and statistically analyzed using $t$-test and multiple testing correction (Benjamini and Hochberg False Discovery Rate). Cut-offs were set twofold and $P \leq 0.01$. Functional clustering of more than twofold differentially expressed genes revealed their regulation. Sets of co-regulated genes identified from microarray analysis were analyzed using WEBbased Gene Set Analysis Toolkit at bioinfo.vanderbilt.edu/webgestalt to predict molecular interaction networks.

\section{Quantitative RT-PCR (qRT-PCR)}

Total RNA was extracted with the RNeasy Mini Kit (Qiagen). After DNase I treatment, $1 \mu \mathrm{g}$ RNA was reverse transcribed using Omniscript RT (Qiagen) and Oligo-dT primer. The cDNA sample was mixed with iQ SYBR Green Supermix (Bio-Rad). QRT-PCR was performed on iQ5 Multicolor Real-Time PCR Detection System (Bio-Rad) using 1/20 volume of the RT reaction. Relative gene expression was calculated using iQ5 Optical System Software. All specific primer pairs used are listed in Supplementary material.

\section{Tumor histology and immunohistochemistry}

Tumor specimens $(\sim \varnothing 8 \mathrm{~mm})$ were removed and fixed in $4 \%$ formaldehyde for at least $48 \mathrm{~h}$. The tissue was dehydrated, defatted, and paraffinized using a histokinette (Leica, Germany). Poured paraffin blocks were cut in $4 \mu \mathrm{m}$ thick sections. Sections were backed for $1 \mathrm{~h}$ on glass slides and processed for either histology by conventionally staining with hemotoxylin/eosin or immunohistochemistry using a BondMaxx Immunostainer (Menarini, Berlin, Germany) with antibodies against pan Granzyme (clone N-19, sc1969, Santa Cruz, CA, USA) and Perforin 1 (clone H-315, sc-9105, Santa Cruz). Slides were dewaxed, pretreated with EDTA for 20s, and incubated with the primary antibodies (dilution 1:400). The pan Granzyme antibody was additionally incubated with an antigoat IgG H\&L antibody (dilution 1:1000, Rockland Inc., Gilbertsville, PA, USA). Immunostaining was visualized using a Mixed-DAB-refine Kit (Menarini) and counterstained with hematoxylin. Slides were scanned and analyzed with a MIXAX slide scanner (Zeiss, Germany).

\section{Results}

\section{Differential gene expression in tumors induced by individual subtype-specific Ret mutations}

To investigate the mechanisms that represent the phenotypic (neoplastic) differences among the MEN2 syndromes, we studied gene expression profiles in growing tumors established by NIH-3T3 cell lines stably expressing specific RET-MEN2A (C609Y, C634R), MEN2B (A883F, M9187) and -FMTC (Y791F) mutations. All NIH-RET transfectants have been extensively characterized for their oncogenic properties in vivo and in cell culture compared with parental NIH-3T3 fibroblasts prior to injection (Miše et al. 2006; data not shown). Our main concern was to find genotype-associated molecular signatures that could explain the earlier onset and aggressiveness of MEN2B-RET-related MTCs. Three independent, whole-genome Affymetrix GeneChip array analyses were performed on each mutation. On the basis of the selection criteria for genes differentially regulated in MEN2B versus MEN2A/FMTC tumors, 1494 genes were obtained, 866 that were upregulated, and 628 that were downregulated in MEN2B (twofold, $P \leq 0.01$ ). By contrast, no significant alterations in gene expression profiles were identified between two varying Ret mutations within individual MEN2A (C609Y versus C634R) or MEN2B (A883F versus M918T) subtypes, suggesting a mechanistic similarity of MEN2 subtype-associated RET mutations. Apart from a low genotypic intra-subtype variability, major differences in gene expression were also not found in MEN2A (C609Y and C634R) versus FMTC tumor samples. Both MEN2A mutations are located in the extracellular cysteine-rich domain of the Ret receptor, whereas the FMTC mutation at codon 791 is found in the cysteine-rich region as well as in the intracellular tyrosine kinase domain. Based on clinical observations, a codon 791 mutation can lead to both FMTC and MEN2A phenotypes, suggesting common mechanisms that are, corresponding to our gene expression data, responsible for the development of both syndromes.

We also performed hierarchical clustering of the microarray data to exhibit the reproducible differential gene expression patterns among MEN2B (A883F+ M918T) versus MEN2A (C609Y + C634R) and FMTC (Y791F) subtype mutations. As shown in Fig. 1, genes with similar expression levels are clustered as homogeneous subgroups. To retrieve functional information on differentially expressed genes in our samples, sets of co-regulated genes (up- or downregulated in MEN2B versus MEN2A/FMTC) 


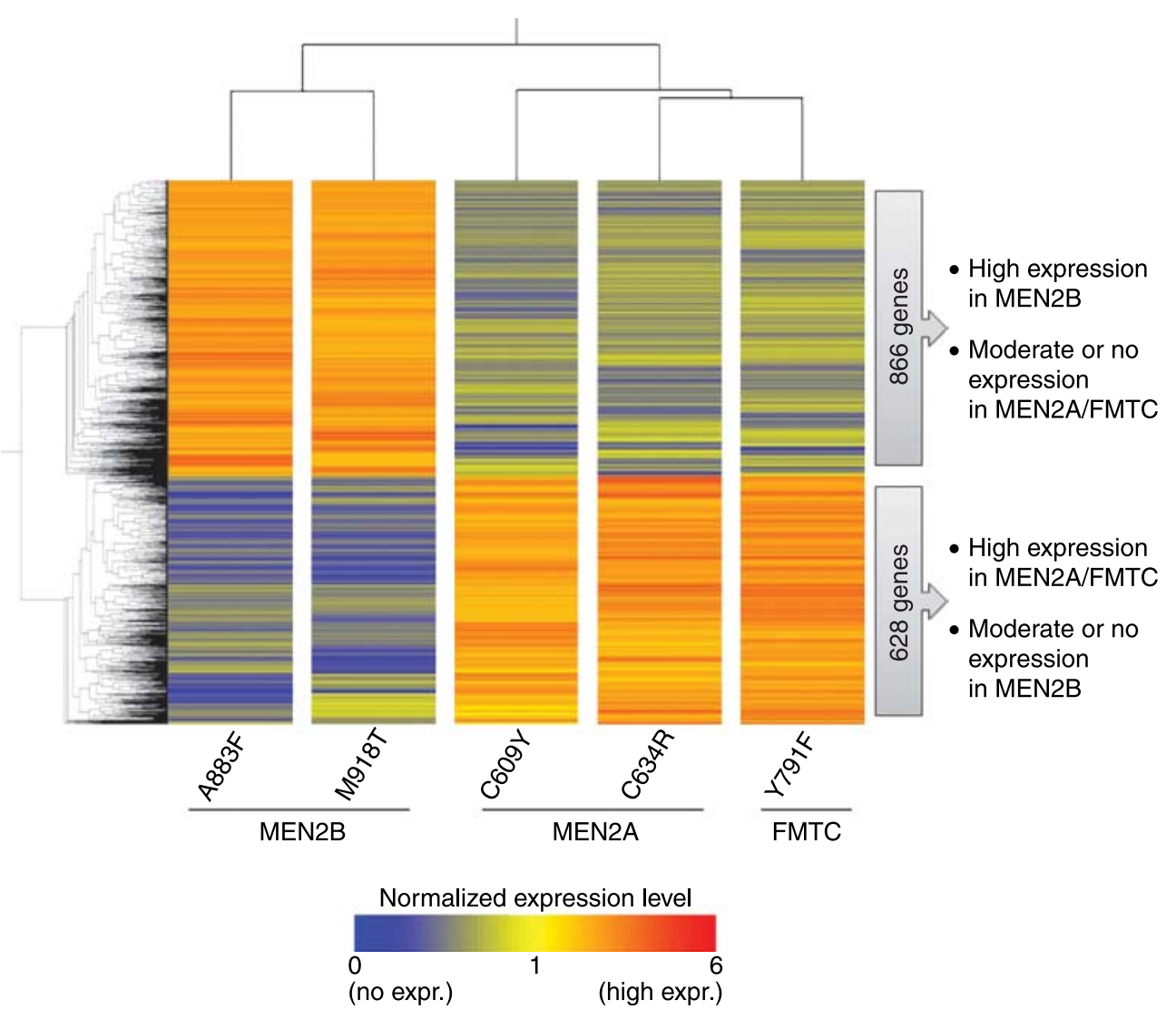

Figure 1 Hierarchical clustering of differentially expressed genes identified by whole-genome gene chip analysis on $\mathrm{NIH}$ RET(MEN2A) (C609Y, C634R), NIH-RET(MEN2B) (A883F, M918T) and NIH-RET(FMTC) (Y791F)-specific tumors. Genes are colored according to the normalized expression values. The colors in the heat map represent expression levels (red, overexpression; yellow, average expression; blue, underexpression/no expression). The tumor samples are ordered in columns and the corresponding gene clusters in rows.

identified from GeneChip arrays were further classified according to their biological process profiles using their Gene Ontology (GO) annotation. Biological processes differentially regulated between MEN2B and MEN2A/FMTC tumors are shown as Supplementary data.

Among the MEN2B tumor-specific gene clusters (shown in Supplementary Table 1, which can be viewed online at http://erc.endocrinology-journals.org/supplemental/) are subclusters enriched of genes involved in cell growth via activation of the phosphatitylinositol 3-kinase (PIK3)/AKT signaling pathway such as related RAS viral (r-ras) oncogene homolog 2 (Rras2) or IGF 2 (Igf2), the CCRK/MAPK3 mitogen-activated protein kinase (MAPK) pathway (Fgf7, Fgfr2, Kras, Map $3 k 8$, Mapkapk3, Ngfb, Prkca, Rps6ka2, Rps6ka6, Rras2, and Grb10), and the WNT signaling pathway with aberrantly activated frizzled homolog $2(F z d 2)$, disheveled associated activator of morphogenesis 2 (Daam2), calcium/calmodulin-dependent protein kinase II $\beta$
(Camk2b), and dickkopf (DKK) homolog Dkk2 and $D k k 3$. Moreover, a large number of genes related to cell cycle progression and oncogenesis (e.g. cyclin E, Ccne1; forkhead box M1, Foxm1) including several growth factors (Ecgf1, Fgf7, Gdf5, Gfer, Hgf, Igf2, $\mathrm{Ng} f$, and $\mathrm{Met}$ ) were found significantly upregulated. Another subcluster contains genes engaged in cell adhesion and metastasis (Col4a6, Lama4, Itga3, Itga6, Itgb4, Cdh2, Foxc2, Hgf, Met, Pcdhb3, and Twist2). In addition, we observed increased expression of various genes in RET-MEN2B specific neoplastic tissues that contribute to cell survival by inducing apoptosis resistance (Ndn, Aatf, Naip2, Traf4, Traf6).

In our attempt to identify biological processes that could discriminate MEN type 2B from 2A/FMTC malignancies, clusters are of particular interest that contain a considerable number of genes involved in the immune system. A total of 56 genes that are expressed at high levels in MEN2A/FMTC tumors and moderately or not in the MEN2B phenotype belonged to the 
host immune and inflammation response category (Supplementary Table 2, which can be viewed online at http://erc.endocrinology-journals.org/supplemental/). Ten of these genes are expressed by natural killer (NK) cells (Cd244, Klra3, Klra12, Klrb1d, Klrc2, Klrd1, Klrel, Ncaml, Ncrl, and Nkg7), others are present on the surface of cytotoxic T lymphocytes (CTLs; for example $C d 28, C d 69, C t l a 4)$. Activated CTLs and NK cells are known to induce lethal damage on their target cells by granule exocytosis or the Fas ligand/Fas system (Henkart 1994, Kojima et al. 1994). In support of a potential NK- and/or T-cell-mediated antitumor immune response to RET/FMTC expressing cells, a number of genes that are highly upregulated in RET/FMTC tumors code for apoptosis molecules such as Fas ligand (FasL), perforin 1 (Prfl), and the majority of murine granzymes (Gzma, Gzmb, Gzmc, Gzmd, Gzme, Gzmf, Gzmg, Gzmk, Gzmn). Seven genes encode cytokines, cytokine receptors, or chemokines (IL15, Il2rb, Il2rg, Ill2rb1, Ill8rap, Icos, Vav3, and $\mathrm{Cx} 3 \mathrm{cll}$ ) critically involved in the stimulation of T- and NK-cell proliferation and their recruitment in cancerous tissues (Allavena et al. 1997, Kuniyasu et al. 2001, Dimberg et al. 2007, Zeng et al. 2007). Ten upregulated genes in this category code for signaling molecules (e.g. Gbp2, Gbp4, interferon-activated genes Ifi202b, Ifi2003, Ifi204, Ifi205, and interferon-produced proteins Ifi44, Ifit3, Ifitml, and Irf8). Consistent with the chemotactic effect of IL15 on T and NK cells by stimulating their adhesion to vascular endothelium (Allavena et al. 1997), cell adhesion molecules such as Vcaml showed enhanced expression in RET-MEN2A/ FMTC tumors.

A pattern of genes related to cell adhesion and/or metastasis was also found upregulated in MEN type 2A/FMTC tumors (Col4a5, Dsc2, Fblim1, Itgal, Itga10, Itgb3, Itgb5, Mmp9, Mmp16, Dmp1, Tgfb2, Postn, Thbs3). Finally, we identified a subcluster enriched of genes that are engaged in skeletal development such as bone morphogenic proteins (BMPs)/receptors (Bmpl, Bmp5, Bmprlb, and Bmpr2), extracellular matrix components (Colloal, Col5a2, and $M g p$ ), members of the transforming growth factor $\beta$-receptor signaling (TGF $\beta$ ) pathway (Bmpr2, Bmp5, Tgfb2), or TGF $\beta$ family binding proteins (Htral, Ltbp4) with higher expression in RET-MEN2A/FMTC than in RET-MEN2B tumors, while six genes that negatively regulate this pathway were upregulated in MEN2B compared with MEN2A/FMTC tissues (Bambi, Eid2, Foxg1, Fst, Fstl3, and Nbll). Members of the TGF $\beta /$ BMP pathways regulate many processes including cellular proliferation, adhesion and differentiation, hematopoiesis, inflammation, wound repair, and skeletal development. The BMP and activin membrane-bound inhibitor, for instance, was identified as a target of the cancer-promoting WNT pathway also upregulated in the RET-MEN2B tumors (Sekiya et al. 2004). However, since MEN2B patients in addition to the early development of invasive tumors frequently develop skeletal abnormalities, the relative lack of TGF $/$ BMP genes in MEN type 2B versus RETMEN2A/FMTC tumors may account for the bone defects as well. By contrast, one example of a factor implicated in bone development that is aberrantly expressed in RET-MEN2B induced tumors is GDF5. In fact, inactivating mutations in this gene are associated with numerous alterations in the length and number of bones in the limbs (Thomas et al. 1996). According to the potential role of $\mathrm{WNT} / \beta$-catenin signaling in new bone formation by functioning as a positive regulator of osteoblasts, and negative regulator of osteoblastdependent osteoclastogenesis (Day et al. 2005, Hu et al. 2005), it appears interesting that secreted WNT inhibitors from the DKK family enhance the osteoclastogenic potency of BMP-driven osteoprogenitors (Fujita \& Janz 2007), whereas the DAAM2 transduces WNT/PCP signals to the RhoA signaling cascade, resulting in the induction of actin skeletal re-organization and cell movement (Mlodzik 2002). These observations suggest that aberrant $D k k 2, D k k 3$, and Daam2 expression in RET-MEN2B cells could cause abnormalities in developing bones that lead to skeletal deformities in patients.

\section{Evaluation of microarray profiles in RET-MEN2- specific tumors and NIH-RET cell cultures in vitro by qRT-PCR}

To further validate the microarray profiles, qPCR confirmation was performed for a representative choice of genes from various functional clusters (Fig. 2) including those coding for proteins involved in the host immune response (Fig. 3) for each Ret mutation using gene-specific primers. QPCR data are illustrated as fold change of expression referred to the lowest gene expression of an individual target. About 32 out of $39(\sim 80 \%)$ showed a consistent change between the microarray and the qPCR results. In 24 of these, the change detected by qPCR was significantly greater, indicating that the statistical criteria applied to the microarray data succeeded in minimizing false positive results.

Quantitative PCR analysis revealed that the mRNAs for proteins involved in the host immune response by the stimulation of NK- and T-cell proliferation, migration, 

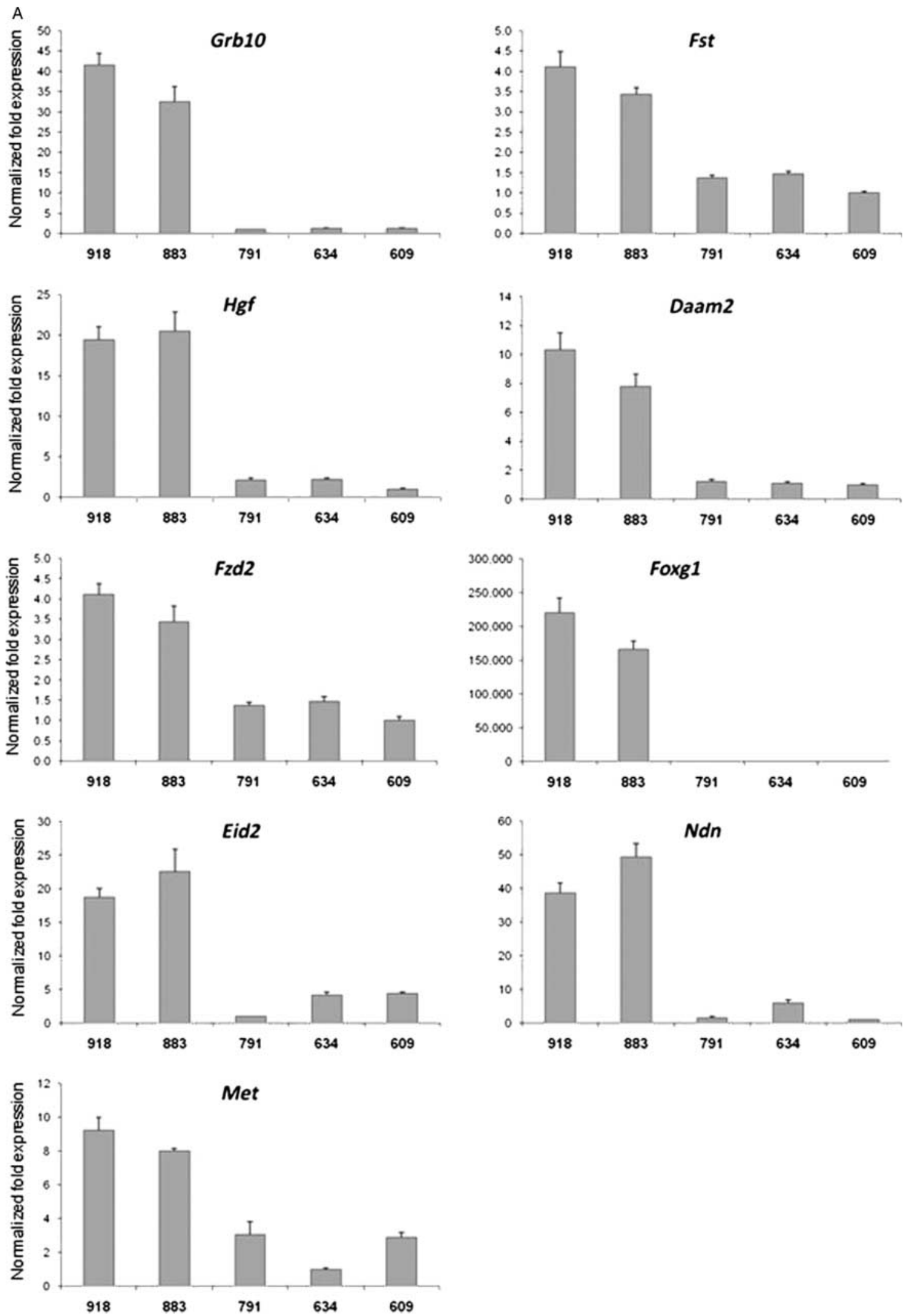

Figure 2 (continued) 

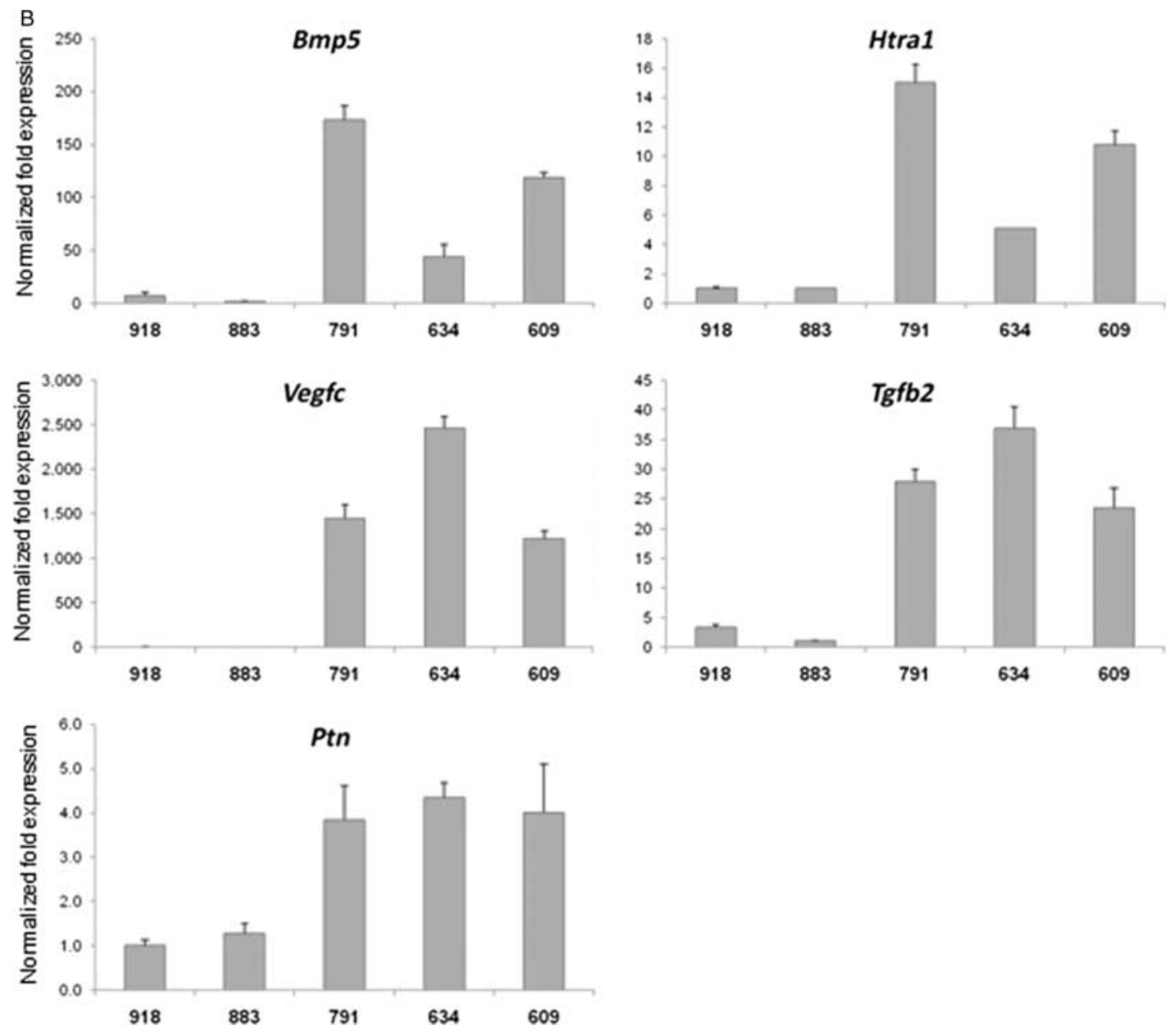

Figure 2 Expression of selected differentially regulated genes in RET-mutation-specific tumors by quantitative RT-PCR analysis. Gene expression levels are shown for individual RET mutations: (A) upregulation in RET-MEN2B 918, 883; and (B) upregulation in RET-MEN2A/FMTC 634, 609/791-related tumors. Fold expression was calculated after normalization with GAPDH and ACTB using iQ5 Multicolor Real-Time PCR Detection System. QPCR data are illustrated as fold change of expression referred to the lowest gene expression of each target (set as 1).

and apoptosis induction of their target cells were highly upregulated in MEN2A/FMTC tumor tissues. By contrast, low or negligible mRNA expression was detected in mouse tumors induced by RET-MEN2B mutations (Fig. 3, grey bars). To clarify which cells are responsible for the expression of the immunomodulatory and death-inducing factors, qRT-PCR analysis was carried out in cultured NIH-RET cell lines harboring these mutations in the absence of the immune system. In fact, aberrant transcript levels of virtually all molecules tested (Gzmb, Gzmf, Prf1, Fasl, Cd244, Nkg7, Il15, $I l 2 r b$, and $N c r l$ ) were detected only in in vivo growing MEN2A/FMTC tumors, but were either absent or expressed at very low levels (Fasl, IL15, Cd244, and $\mathrm{Nkg} 7$ ) in cell cultures, suggesting that they are attributed to activated infiltrating immune effector cells such as NK cells and T lymphocytes. Conversely, fractalkine
(Cx3cll) considered as a chemokine involved in the recruitment and accumulation of leukocytes into cancerous tissues is also expressed at comparable high levels in MEN2A/FMTC-transformed cell lines. Importantly, this chemokine is not expressed in both RETMEN2B tumor specimens.

To substantiate the assumption of enhanced infiltration of immune cells into MEN type 2A/FMTC compared with RET-MEN2B tumors, we performed immunohistochemical examination of Ret genotypespecific tumor sections using antibodies specific against pan granzyme and perforin 1, exclusively expressed by NK cells and CTLs as the major mechanism by which these cells exert an immune response on tumor cells (Kawasaki et al. 1992, Grossman et al. 2003). As shown in Fig. 4, inflammatory infiltrates were detected only in 

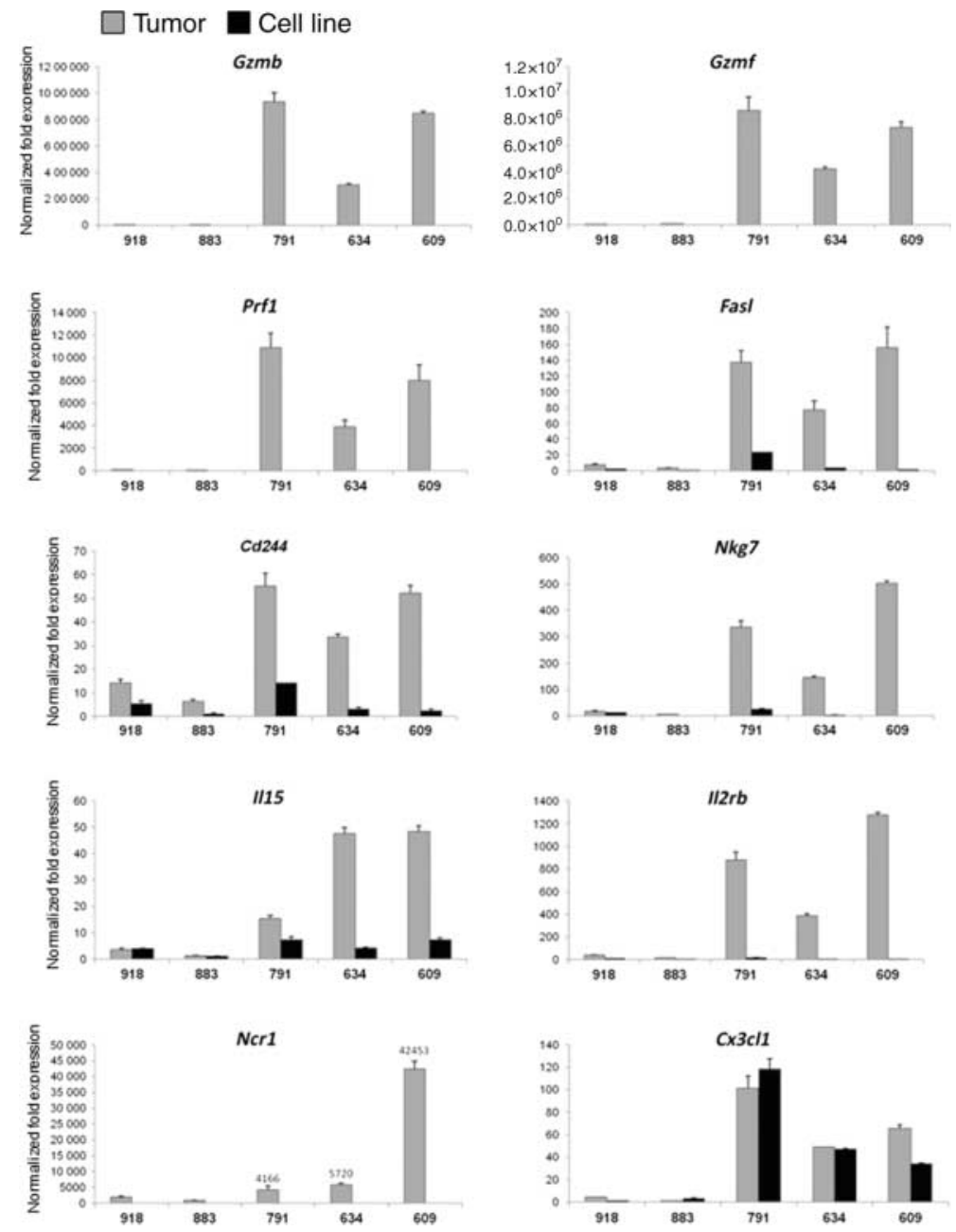

Figure 3 Differential expression of genes involved in host immune response and recruitment of immune effector cells in RETMEN2A/FMTC versus RET-MEN2B. Selected immune response genes (Gzmb, Gzmf, Prf1, Fasl, I/15, and II2rb), NK cell markers (Cd244, Nkg7, and Ncr1) and chemokines (Cx3cl1) were verified in established RET-specific tumors (grey bars) and cultured NIHRET cell lines (black bars) by QPCR. Data are illustrated as fold change of expression after normalization with GAPDH and ACTB using iQ5 Multicolor Real-Time PCR Detection System. The lowest gene expression of each target was set as 1.

RET-MEN2A/FMTC-induced tumors as indicated by a strong granzyme/perforin staining (brown cells). By contrast, there was no detectable immunoreactivity in RET-MEN2B tissues indicative for the complete lack of inflammatory infiltrates in these tumors. NIH-RET tumor cells by itself stained negative. We also attempted to detect fractalkine using anti-CX3CL1 antibody (NEUROMICS) in tumor sections. Immunohistochemical staining showed that fractalkine immunoreactivity was mainly observed in single extracellular conglomerates, suggesting that the chemokine is present in its soluble (secreted) form (data not shown). This is in agreement with a higher expression of the tumor necrosis factor- $\alpha$-converting enzyme (ADAM17) in MEN2A/FMTC tumors, inducing the release of CX3CL1 through its cleavage at a membrane-proximal site.

\section{Discussion}

We compared the whole-genome expression profiles triggered by specific RET-MEN2A, -B and FMTC mutations using a defined in vivo model of growing NIH-RET-related tumors in mice. Based on the selection criteria for up- and downregulated expressions in MEN2B versus MEN2A/ FMTC, 1494 differential genes were observed. Apart from a low genotypic intra-subtype variability, surprisingly no significant alterations in gene expression were found in MEN2A versus FMTC species. A possible 


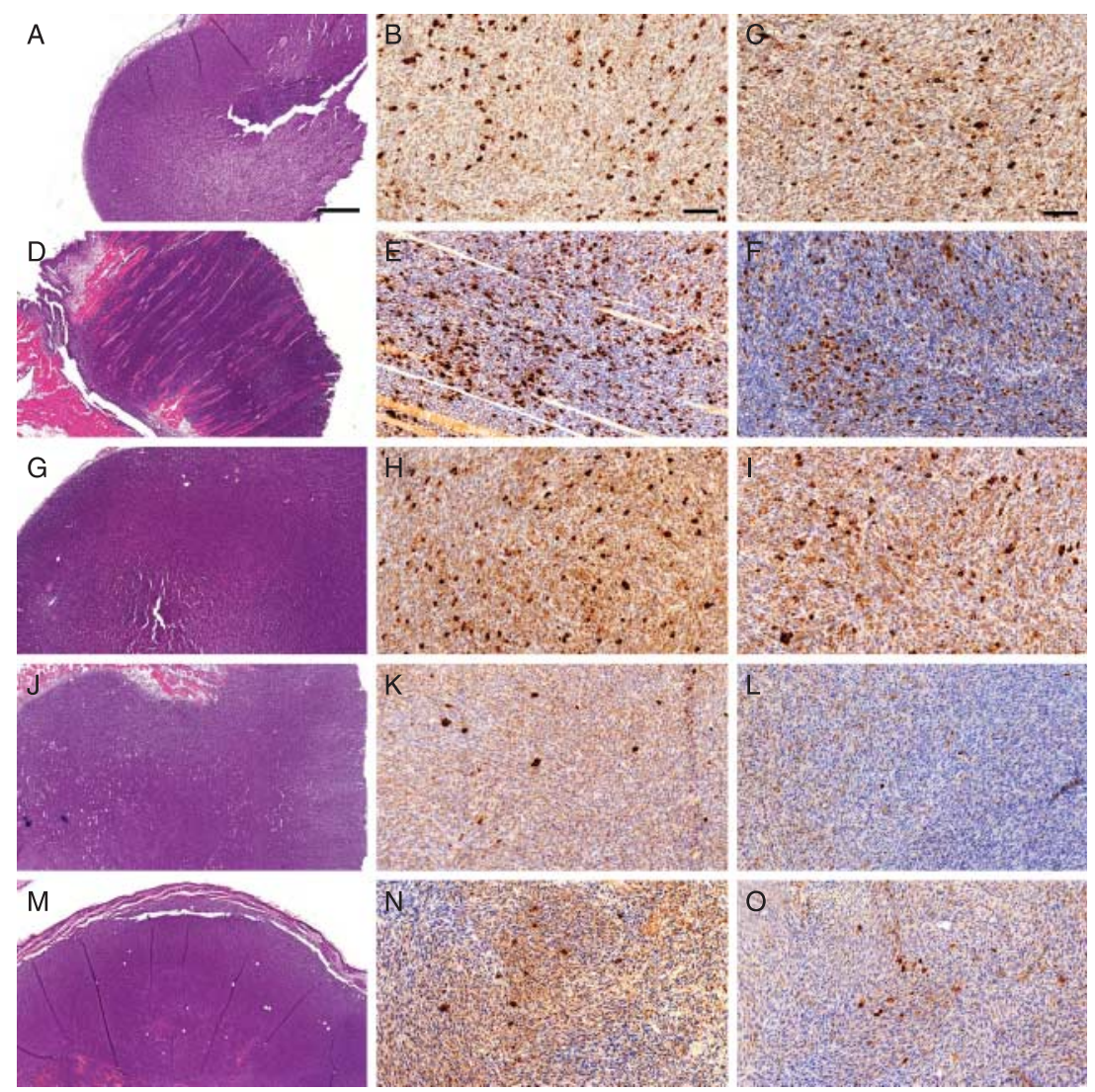

Figure 4 Verification of inflammatory infiltrates in RET-MEN2A/FMTC versus RET-MEN2B-induced tumors by immunohistochemistry. Paraffin sections of subcutaneously established NIH-RET tumor carrying MEN2A-C634R (A-C), MEN2A-C609Y (D-F), FMTC-Y791F (G-I), MEN2B-M918T (J-L), and MEN2B-A883F (M-O) mutations. Immune cells were detected with antibodies specific for pan-granzyme (middle column; B-N) and perforin 1 (right column; C-O). A strong positive reaction (brown cells) was observed in section of RET-MEN2A/FMTC specimens. Hematoxylin and eosin (HE) staining (left column: A-M). Original magnification $\times 5$, scale bar $=500 \mu \mathrm{m}(\mathrm{HE}) ; \times 20$, scale bar $=100 \mu \mathrm{m}$ (pan-granzyme/perforin 1$)$.

explanation is that a codon 791 mutation can lead to FMTC and MEN2A phenotypes, suggesting a common mechanism responsible for the development of both syndromes. Whether patients with a mutation at codon 791 have a high or least high risk for aggressive MTC presumably depends on additional perhaps environmental conditions. Our findings, however, indicate that both genotypes per se exhibit a similar oncogenic potential. Instead, GO classification and TreeView analysis of discriminating gene clusters in MEN type 2B and RET-MEN2A/FMTC-induced tumors showed obvious differences in characteristic survival pathways and, in particular, the host antitumor immune response between both cancer types.

\section{MEN2B-specific tumors are characterized by individual survival pathways}

Transformation by MEN2B-RET is potentially a consequence of aberrant activation of signaling pathways that are normally regulated by cytoplasmic tyrosine kinases. We found that several genes that are important for RAS/MAPK or PIK3/AKT signaling are expressed at higher levels in MEN2B than in the tumors expressing MEN2A /FMTC-RET. Members of these pathways are essential mediators of cellular responses to extracellular signals that include growth factors, hormones, cytokines, and environmental stress (Chang \& Karin 2001), which regulate essentially all aspects of malignant cell behavior, proliferation, survival, migration, and invasion. Signaling cascades such as the RAS/MAPK or PIK3/AKT pathway are activated mainly via phosphorylated tyrosine 1062 in RET (Salvatore et al. 2001). In line with this, phosphorylation of RET at Y1062 has been shown stronger in RET-MEN2B than in RET-MEN2A or FMTC expressing cells (Miše et al. 2006). However, while cancer is most often thought of as a disease that is caused by inappropriate levels of proliferation, apoptosis shares an equally if not more important 
role in cancer progression and metastasis. As an antiapoptotic pathway, the mitogenic MAPK cascade is characterized by the sequential activation of the RAF, MAP3K1, MAPK1, and RPS6KA2/RPS6KA kinases (Bonni et al. 1999). MAPK-mediated survival also involves proteins of the growth factor receptor-bound (Grb) adapter family Grb7/10/14 that bind to the RET tyrosine kinase receptor. In particular, Raf1 and Grb10 have been shown to be required for the ability of the MAP kinase pathway to modulate the phosphorylation and inactivation of Bad, thereby creating a specific antiapoptotic activity (Kebache et al. 2007). Interestingly, our analysis revealed an upregulation of both Grb10/Grb14 and Rps6ka kinases in MEN2B species, which together with their previously noticed increased apoptosis resistance (Miše et al. 2006), may reflect the MAPK prosurvival function in these tumors. Consistent with the assumption that apoptosis suppressive activities in MEN2B versus MEN2A/FMTC cases likely contribute to disease aggessiveness, additional cell survival-related transcripts were found considerably expressed in RET-918 and RET-883 tumors. In particular, growing evidence has demonstrated that the hepatocyte growth factor (HGF) and Met protein overexpressed in many human cancers including oncogene-transformed human thyroid cells (Eccles et al. 1996) play a major role in tumor progression, invasion, and metastasis (Jeffers et al. 1996). Papillary thyroid carcinoma in which both components are activated in an autocrine fashion (Trovato et al. 1998) are characterized by highly invasive behavior and early metastatic spread (Ruco et al. 2001). Since Ret can directly induce overexpression of Met in human thyroid epithelial cells (Ivan et al. 1997), an intriguing possibility to explain the higher aggressiveness of the MEN2B phenotype is that activated RET-MEN2B mutations and the HGF-Met signal act in a synergistic manner at the early stage of tumor development, likely by converging on a common target such as the RAS/ MAPK or PIK3/AKT cascades, thereby providing an extra growth advantage over cells expressing the MEN2A/FMTC mitogenic RET signal. Because suspension-induced apoptosis (anoikis) resistance plays an important role in tumor progression and metastasis, it is of interest that HGF provides protection against anoikis through activation of MAPK and AKT signaling pathways in head and neck squamous cell carcinoma (Zeng et al. 2002).

Additional significance that apoptosis resistance plays an essential role in RET-MEN2B tumor progression is provided by the upregulation of TWIST2. As one of the E-cadherin repressors, TWIST regulates the epithelial-to-mesenchymal transition and promotes tumor metastasis (Yang et al. 2004). TWIST2 antagonizes TRP53-induced apoptosis (Maestro et al. 1999). Another protein whose expression is particularly high in the RET-MEN2B tumors that potentially confers resistance to apoptosis is neurally differentiated embryonal carcinoma cellderived factor (necdin). Previous results have shown that this protein blocks E2F1-induced apoptosis in neuroblastoma cells (Kobayashi et al. 2002). Moreover, necdin interacts with the transactivation domain of p53, thereby inhibiting p53-mediated apoptosis of osteosarcoma cells (Taniura et al. 1999). Thus, identifying the endogenous binding proteins of necdin in cells expressing RET mutations is likely to shed light on the molecular basis for a putative apoptosis inhibitory function in these cells and how this may contribute to enhanced tumor aggressiveness.

\section{Dysregulation of host antitumor response mechanisms during RET-MEN type 2B tumorigenesis}

A central finding of this study was the extent of changes in genes whose products affect the immune response. In particular, we observed a remarkable accumulation of genes encoding NK cell receptors, T-lymphocyte antigens, regulators of NK- and T-cell proliferation/attraction, and apoptosis molecules important for the ability of NK cells and cytotoxic T cells to kill their targets in the tumors initiated by RETMEN type 2A/FMTC mutations, while expression of these genes was nearly completely suppressed in both RET-MEN2B species. A comparative analysis of the transcript levels in transplanted tumor tissues and in cultured NIH-3T3 cell lines harboring single Ret mutations revealed that the majority of immunomodulatory genes are exclusively upregulated in mice growing tumors, indicating that these molecules are attributed to the host innate immune system. In support of this notion, we found massive inflammatory infiltrates in sections from RET-MEN2A/FMTC tumors as evidenced by a positive perforin and pan granzyme staining that were barely detectable in neoplastic tissues of RET-MEN2B origin. The strong representation of immune- and inflammation responserelated genes in RET-MEN2A/FMTC-induced tumors and their apparent lack in more aggressive RET malignancies points to a critical perhaps differential role of RET oncoproteins in the early modulation of immune response mechanisms. Tumor-infiltrating leukocytes can negatively regulate tumor progression by producing cytostatic or cytotoxic molecules. At the molecular level, the granule exocytosis pathway and 


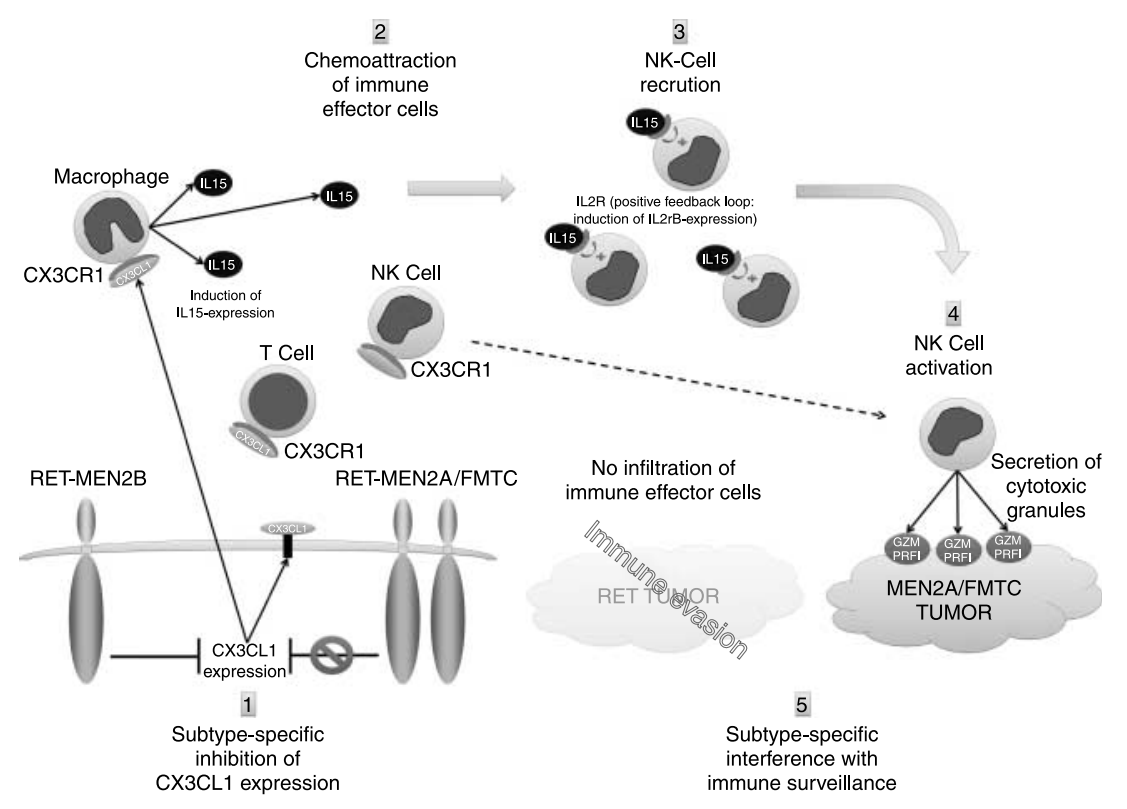

Figure 5 Model of RET receptor subtype-specific interference with the innate immune system of nude mice. RET-MEN2A/FMTCtransformed cells produce CX3CL1 (fractalkine), whereas cells induced by RET-MEN2B mutations anticipate chemokine expression (1). The secreted membrane-bound and soluble forms of CX3CL1 cause recruitment (chemotaxis and adhesion) of NK cells, cytotoxic lymphocytes, and monocyte-macrophages expressing its receptor CX3CR1 (2). IL15 produced by activated monocytemacrophages, for example, attracts T and NK cells to tumor endothelium and stimulates their proliferation via interaction with the IL2 receptor $\beta$ (3). Tumor-infiltrating activated NK and T cells secrete cytotoxic granules (4) that potentially leads to enhanced killing of MEN type 2A/FMTC-specific tumors, while RET-MEN2B receptors avoid immune infiltration as a mechanism of evasion (5).

Fas-FasL system account for virtually all of the measurable contact-mediated cytotoxicity delivered by NK cells and CD8 + T cells (Russell \& Ley 2002, Lieberman 2003). The granzyme pathway utilizes perforin to traffic granzymes appropriately into the cytosol of target cells (Shi et al. 1997, Keefe et al. 2005), where granzymes A and B (and orphan granzymes) induce cell death by clearing critical substrates (Russell \& Ley 2002, Lieberman 2003). Thus far, in mouse experimental tumor systems, perforin activities have been demonstrated to protect the host against tumor initiation (van den Broek et al. 1996), primary tumor growth (van den Broek et al. 1996, Smyth et al. 1998), and tumor metastasis (Smyth et al. 1999). Intriguingly, in our transplanted model, nearly all mouse granzyme encoding genes (A, B, C, D, $\mathrm{E}, \mathrm{F}, \mathrm{G}, \mathrm{K}$, and $\mathrm{N}$; Grossman et al. 2003) are extensively expressed in the RET-MEN2A/ FMTC-related tumors, suggesting that this immune surveillance system may operate at early stages of tumorigenesis, possibly leading to tumor growth control. This assumption is substantiated by our previous findings, showing that neoplastic cell growth of faster proliferating NIH-RET mutants associated with MEN2A and FMTC is slowed down shortly after tumor cell transplantation into nude mice, and can then be overcome by basically less proliferative RETMEN2B expressing cells (Miše et al. 2006). The observed growth behavior of RET-MEN2A/FMTC specimens may be due to the formation of an effective antitumor immune response that eventually increases susceptibility to NK- and T-cell lysis, thereby contributing to growth control, and perhaps a less aggressive phenotype. In fact, cytotoxic T- and tumorinfiltrating NK cells have been associated with a favorable prognosis in a variety of human malignancies (Coca et al. 1997, Ishigami et al. 2000, Takanami et al. 2001, Hsia et al. 2005).

The data herein suggest that, whereas RET-MEN2A and FMTC mutations are related to antitumor immunity, oncogenic receptor tyrosine kinase signaling specifically initiated by MEN type $2 \mathrm{~B}$ mutations causally determines immune defense. In agreement with a contributory role of MEN2A/FMTC in cancerassociated inflammation, NIH-3T3 transfectants with these mutations show a significantly enhanced expression of Cx3cll both in vitro and when transplanted into mice. This chemokine is critically important for infiltration (chemotaxis and adhesion) of various lineages of lymphocytes characterized by a high content of intracellular perforin and granzyme, and monocytes/macrophages expressing its receptor 
CX3CR1 in human carcinomas (Guo et al. 2003, Ohta et al. 2005). Previous reports have demonstrated that the antitumor effects elicited by fractalkine gene transfer into subcutaneously implanted murine cancer cell lines depend on NK and T cells (Guo et al. 2003, Lavergne et al. 2003, Xin et al. 2005). Consistent with our data, high endogenous fractalkine expression in neuroblastoma (Zeng et al. 2007), colorectal cancer (Ohta et al. 2005), and gastric adenocarcinoma (Hyakudomi et al. 2008), where it is mainly observed in the plasma membrane and cytoplasm of tumor cells, correlated with a higher density of tumor-infiltrating immune cells. In case of RET oncogene-induced tumors, this activation is dependent on the kind of RET mutation, since fractalkine is not expressed in MEN2B tumors lacking immune infiltrates. The exact mechanism by which MEN type 2A/FMTC RET maintains chemokine production, or more relevant, RET-MEN2B counteracts immune infiltration by anticipating CX3CL1 expression is presently unknown and subject to further investigations. It was reported that $\mathrm{Cx} 3 \mathrm{cll}$ mRNA and protein expressions are induced by various inflammatory stimuli including IL1, TNF- $\alpha$ and IFNG in endothelial cells (Fraticelli et al. 2001), but these factors were not found differentially regulated in RET-MEN2A/FMTC versus RET-MEN2B tumors, implicating that other oncogene-related mechanisms may account for the effect. Together with the studies demonstrating that a high expression of fractalkine in cancer cells drastically reduce their metastatic potential, thus resulting in a better prognosis for patients (Ohta et al. 2005, Vitale et al. 2007), this phenomenon raises the possibility of increased tumor cell killing in MEN2A/FMTC specimens, and may explain the faster progression of MEN2B tumors to clinically significant forms by a RET-MEN2B receptor-specific prevention of molecules involved in recruitment of $\mathrm{T}$ - and/or NK-cell cytotoxicity, thereby allowing developing cancer cells to grow unchecked in the absence of otherwise intact host immune responses.

So far, a differential display analysis of NIH3T3 fibroblasts transfected with RET-MEN2A mutation 634 and MEN2B mutation 918 has been reported (Watanabe et al. 2002). In addition, expression analysis of tumors from MEN2A and MEN2B patients was performed (Jain et al. 2004). Although, we identified a much larger spectrum of differentially expressed genes, data from the present approach are generally consistent with these studies, indicating that genes involved in the process of cell growth and epithelial to mesenchymal transition are characteristic for MEN2A and MEN2B tumors respectively. For single targets such as pleiotropin, which is specifically upregulated in MEN2A compared with MEN2B tumors (Watanabe et al. 2002), we obtained similar results using a different technology. The differences in genes identified to their experiments including those genes associated with the host immune response presumably reflect our in vivo versus in vitro design or the mouse versus human expression profiling of tumors at an early stage of development.

In summary, our data support a model of Ret oncogene-specific interference with the host immune system (Fig. 5), in which chemokine production by RET-MEN2A/FMTC cancer cells initiates an antitumor immune attack, while RET-MEN2B receptors avoid tumor infiltration as a mechanism of evasion that may be critical for the different clinical outcome of both subtypes.

\section{Declaration of interest}

The authors declare that there is no conflict of interest that would prejudice the impartiality of this scientific work.

\section{Funding}

This work was supported by DFG grant PU 188/3-3/3-4 (B M Pützer).

\section{Acknowledgements}

We thank B Müller-Hilke for helpful discussion.

\section{References}

Allavena P, Giardina G, Bianchi G \& Mantovani A 1997 IL-15 is chemotactic for natural killer cells and stimulates their adhesion to vascular endothelium. Journal of Leukocyte Biology 61 729-735.

Bonni A, Brunet A, West AE, Datta SR, Takasu MA \& Greenberg ME 1999 Cell survival promoted by the RasMAPK signaling pathway by transcription-dependent and -independent mechanisms. Science 286 1358-1362.

van den Broek ME, Kagi D, Ossendorp F, Toes R, Vamvakas S, Lutz WK, Melief CJ, Zinkernagel RM \& Hengartner H 1996 Decreased tumor surveillance in perforin-deficient mice. Journal of Experimental Medicine 184 1781-1790.

Chang L \& Karin M 2001 Mammalian MAP kinase signalling cascades. Nature 410 37-40.

Coca S, Perez-Piqueras J, Martinez D, Colmenarejo A, Saez MA, Vallejo C, Martos JA \& Moreno M 1997 The prognostic significance of intratumoral natural killer cells in patients with colorectal carcinoma. Cancer 79 2320-2328. 
Day TF, Guo X, Garrett-Beal L \& Yang Y 2005 Wnt/betacatenin signaling in mesenchymal progenitors controls osteoblast and chondrocyte differentiation during vertebrate skeletogenesis. Developmental Cell 8 739-750.

Dimberg J, Dienus O, Lofgren S, Hugander A \& Wagsater D 2007 Polymorphisms of Fractalkine receptor CX3CR1 and plasma levels of its ligand CX3CL1 in colorectal cancer patients. International Journal of Colorectal Disease 22 1195-1200.

Drosten M \& Pützer BM 2006 Mechanisms of disease: cancer targeting and the impact of oncogenic RET for medullary thyroid carcinoma therapy. Nature Clinical Practice. Oncology 3 564-574.

Eccles N, Ivan M \& Wynford-Thomas D 1996 Mitogenic stimulation of normal and oncogene-transformed human thyroid epithelial cells by hepatocyte growth factor. Molecular and Cellular Endocrinology 117 247-251.

Fraticelli P, Sironi M, Bianchi G, D’ Ambrosio D, Albanesi C, Stoppacciaro A, Chieppa M, Allavena P, Ruco L, Girolomoni G et al. 2001 Fractalkine (CX3CL1) as an amplification circuit of polarized Th1 responses. Journal of Clinical Investigation 107 1173-1181.

Fujita K \& Janz S 2007 Attenuation of WNT signaling by DKK-1 and -2 regulates BMP2-induced osteoblast differentiation and expression of OPG, RANKL and M-CSF. Molecular Cancer 671.

de Groot JW, Links TP, Plukker JT, Lips CJ \& Hofstra RM 2006 RET as a diagnostic and therapeutic target in sporadic and hereditary endocrine tumors. Endocrine Reviews 27 535-560.

Grossman WJ, Revell PA, Lu ZH, Johnson H, Bredemeyer AJ \& Ley TJ 2003 The orphan granzymes of humans and mice. Current Opinion in Immunology 15 544-552.

Guo J, Zhang M, Wang B, Yuan Z, Guo Z, Chen T, Yu Y, Qin Z \& Cao X 2003 Fractalkine transgene induces T-celldependent antitumor immunity through chemoattraction and activation of dendritic cells. International Journal of Cancer 103 212-220.

Henkart PA 1994 Lymphocyte-mediated cytotoxicity: two pathways and multiple effector molecules. Immunity $\mathbf{1}$ 343-346.

Hsia JY, Chen JT, Chen CY, Hsu CP, Miaw J, Huang YS \& Yang CY 2005 Prognostic significance of intratumoral natural killer cells in primary resected esophageal squamous cell carcinoma. Chang Gung Medical Journal 28 335-340.

Hu H, Hilton MJ, Tu X, Yu K, Ornitz DM \& Long F 2005 Sequential roles of Hedgehog and Wnt signaling in osteoblast development. Development 132 49-60.

Hyakudomi M, Matsubara T, Hyakudomi R, Yamamoto T, Kinugasa S, Yamanoi A, Maruyama R \& Tanaka T 2008 Increased expression of fractalkine is correlated with a better prognosis and an increased number of both CD8+ $\mathrm{T}$ cells and natural killer cells in gastric adenocarcinoma. Annals of Surgical Oncology 15 1775-1782.
Ishigami S, Natsugoe S, Tokuda K, Nakajo A, Che X, Iwashige H, Aridome K, Hokita S \& Aikou T 2000 Prognostic value of intratumoral natural killer cells in gastric carcinoma. Cancer 88 577-583.

Ivan M, Bond JA, Prat M, Comoglio PM \& WynfordThomas D 1997 Activated ras and ret oncogenes induce over-expression of c-met (hepatocyte growth factor receptor) in human thyroid epithelial cells. Oncogene 14 2417-2423.

Jain S, Watson MA, DeBenedetti MK, Hiraki Y, Moley JF \& Milbrandt J 2004 Expression profiles provide insights into early malignant potential and skeletal abnormalities in multiple endocrine neoplasia type $2 \mathrm{~B}$ syndrome tumors. Cancer Research 64 3907-3913.

Jeffers M, Rong S \& Woude GF 1996 Hepatocyte growth factor/scatter factor-Met signaling in tumorigenicity and invasion/metastasis. Journal of Molecular Medicine $\mathbf{7 4}$ 505-513.

Kawasaki A, Shinkai Y, Yagita H \& Okumura K 1992 Expression of perforin in murine natural killer cells and cytotoxic T lymphocytes in vivo. European Journal of Immunology 22 1215-1219.

Kebache S, Ash J, Annis MG, Hagan J, Huber M, Hassard J, Stewart CL, Whiteway M \& Nantel A 2007 Grb10 and active Raf-1 kinase promote Bad-dependent cell survival. Journal of Biological Chemistry 28221873 21883.

Keefe D, Shi L, Feske S, Massol R, Navarro F, Kirchhausen T \& Lieberman J 2005 Perforin triggers a plasma membrane-repair response that facilitates CTL induction of apoptosis. Immunity 23 249-262.

Kobayashi M, Taniura H \& Yoshikawa K 2002 Ectopic expression of necdin induces differentiation of mouse neuroblastoma cells. Journal of Biological Chemistry 277 42128-42135.

Kojima H, Shinohara N, Hanaoka S, Someya-Shirota Y, Takagaki Y, Ohno H, Saito T, Katayama T, Yagita H, Okumura K et al. 1994 Two distinct pathways of specific killing revealed by perforin mutant cytotoxic $\mathrm{T}$ lymphocytes. Immunity 1 357-364.

Kuniyasu H, Oue N, Nakae D, Tsutsumi M, Denda A, Tsujiuchi T, Yokozaki H \& Yasui W 2001 Interleukin-15 expression is associated with malignant potential in colon cancer cells. Pathobiology 69 86-95.

Lakhani VT, You YN \& Wells SA 2007 The multiple endocrine neoplasia syndromes. Annual Review of Medicine 58 253-265.

Lavergne E, Combadiere B, Bonduelle O, Iga M, Gao JL, Maho M, Boissonnas A, Murphy PM, Debre P \& Combadiere C 2003 Fractalkine mediates natural killerdependent antitumor responses in vivo. Cancer Research $637468-7474$.

Lieberman J 2003 The ABCs of granule-mediated cytotoxicity: new weapons in the arsenal. Nature Reviews. Immunology 3 361-370. 
Maestro R, Dei Tos AP, Hamamori Y, Krasnokutsky S, Sartorelli V, Kedes L, Doglioni C, Beach DH \& Hannon GJ 1999 Twist is a potential oncogene that inhibits apoptosis. Genes and Development 13 2207-2217.

Miše N, Drosten M, Racek T, Tannapfel A \& Putzer BM 2006 Evaluation of potential mechanisms underlying genotype-phenotype correlations in multiple endocrine neoplasia type 2. Oncogene 25 6637-6647.

Mlodzik M 2002 Planar cell polarization: do the same mechanisms regulate Drosophila tissue polarity and vertebrate gastrulation? Trends in Genetics $\mathbf{1 8}$ 564-571.

Ohta M, Tanaka F, Yamaguchi H, Sadanaga N, Inoue H \& Mori M 2005 The high expression of Fractalkine results in a better prognosis for colorectal cancer patients. International Journal of Oncology 26 41-47.

Plaza-Menacho I, Burzynski GM, de Groot JW, Eggen BJL \& Hofstra RMW 2006 Current concepts in RET-related genetics, signaling and therapeutics. Trends in Genetics 22 627-636.

Ruco LP, Stoppacciaro A, Ballarini F, Prat M \& Scarpino S 2001 Met protein and hepatocyte growth factor (HGF) in papillary carcinoma of the thyroid: evidence for a pathogenetic role in tumourigenesis. Journal of Pathology 194 4-8.

Russell JH \& Ley TJ 2002 Lymphocyte-mediated cytotoxicity. Annual Review of Immunology 20 323-370.

Salvatore D, Melillo RM, Monaco C, Visconti R, Fenzi G, Vecchio G, Fusco A \& Santoro M 2001 Increased in vivo phosphorylation of ret tyrosine 1062 is a potential pathogenetic mechanism of multiple endocrine neoplasia type 2B. Cancer Research 61 1426-1431.

Sekiya T, Adachi S, Kohu K, Yamada T, Higuchi O, Furukawa Y, Nakamura Y, Nakamura T, Tashiro K, Kuhara S et al. 2004 Identification of BMP and activin membrane-bound inhibitor (BAMBI), an inhibitor of transforming growth factor-beta signaling, as a target of the beta-catenin pathway in colorectal tumor cells. Journal of Biological Chemistry 279 6840-6846.

Shi L, Mai S, Israels S, Browne K, Trapani JA \& Greenberg AH 1997 Granzyme B (GraB) autonomously crosses the cell membrane and perforin initiates apoptosis and GraB nuclear localization. Journal of Experimental Medicine 185 855-866.

Smyth MJ, Kershaw MH, Darcy PK \& Trapani JA 1998 Adoptive transfer: the role of perforin in mouse cytotoxic T lymphocyte rejection of human tumor xenografts in vivo. Xenotransplantation 5 146-153.

Smyth MJ, Thia KY, Cretney E, Kelly JM, Snook MB, Forbes CA \& Scalzo AA 1999 Perforin is a major contributor to NK cell control of tumor metastasis. Journal of Immunology 162 6658-6662.
Takahashi M, Ritz J \& Cooper GM 1985 Activation of a novel human transforming gene, ret, by DNA rearrangement. Cell 42 581-588.

Takanami I, Takeuchi K \& Giga M 2001 The prognostic value of natural killer cell infiltration in resected pulmonary adenocarcinoma. Journal of Thoracic and Cardiovascular Surgery 121 1058-1063.

Taniura H, Matsumoto K \& Yoshikawa K 1999 Physical and functional interactions of neuronal growth suppressor necdin with p53. Journal of Biological Chemistry 274 16242-16248.

Thomas JT, Lin K, Nandedkar M, Camargo M, Cervenka J \& Luyten FP 1996 A human chondrodysplasia due to a mutation in a TGF-beta superfamily member. Nature Genetics 12 315-317.

Trovato M, Villari D, Bartolone L, Spinella S, Simone A, Violi MA, Trimarchi F, Batolo D \& Benvenga S 1998 Expression of the hepatocyte growth factor and c-met in normal thyroid, non-neoplastic, and neoplastic nodules. Thyroid 8 125-131.

Vitale S, Cambien B, Karimdjee BF, Barthel R, Staccini P, Luci C, Breittmayer V, Anjuere F, Schmid-Alliana A \& Schmid-Antomarchi H 2007 Tissue-specific differential antitumour effect of molecular forms of fractalkine in a mouse model of metastatic colon cancer. Gut 56 365-372.

Watanabe T, Ichihara M, Hashimoto M, Shimono K, Shimoyama Y, Nagasaka T, Murakumo Y, Murakami H, Sugiura H, Iwata H et al. 2002 Characterization of gene expression induced by RET with MEN2A or MEN2B mutation. American Journal of Pathology 161 249-256.

Xin H, Kikuchi T, Andarini S, Ohkouchi S, Suzuki T, Nukiwa T, Huqun, Hagiwara K, Honjo T \& Saijo Y 2005 Antitumor immune response by CX3CL1 fractalkine gene transfer depends on both NK and T cells. European Journal of Immunology 35 1371-1380.

Yang J, Mani SA, Donaher JL, Ramaswamy S, Itzykson RA, Come C, Savagner P, Gitelman I, Richardson A \& Weinberg RA 2004 Twist, a master regulator of morphogenesis, plays an essential role in tumor metastasis. Cell 117 927-939.

Zeng Q, Chen S, You Z, Yang F, Carey TE, Saims D \& Wang CY 2002 Hepatocyte growth factor inhibits anoikis in head and neck squamous cell carcinoma cells by activation of ERK and Akt signaling independent of NFkappa B. Journal of Biological Chemistry 277 25203-25208.

Zeng Y, Huebener N, Fest S, Weixler S, Schroeder U, Gaedicke G, Xiang R, Schramm A, Eggert A, Reisfeld RA et al. 2007 Fractalkine (CX3CL1)- and interleukin-2enriched neuroblastoma microenvironment induces eradication of metastases mediated by $\mathrm{T}$ cells and natural killer cells. Cancer Research 67 2331-2338. 